
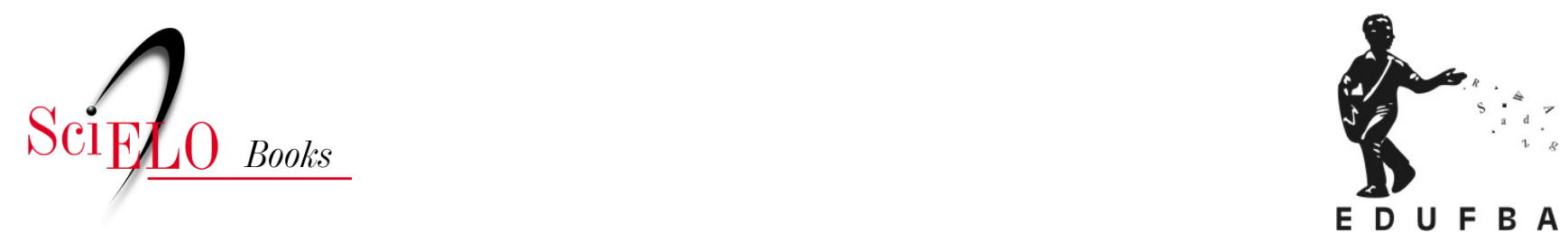

\title{
Comportamento de industriários quanto à prevenção do câncer da próstata
}

\author{
André Luis Santos Virgulino \\ Marco Antônio Vasconcelos Rêgo
}

\section{SciELO Books / SciELO Livros / SciELO Libros}

VIRGULINO, A.L.S., and RÊGO, M.A.V. Comportamento de industriários quanto à prevenção do câncer da próstata. In: FERNANDES, R.C.P., LIMA, M.A.G., and ARAÚJO, T.M., comps. Tópicos em saúde, ambiente e trabalho: um olhar ampliado [online]. Salvador: EDUFBA, 2014, pp. 423-450. ISBN: 978-65-5630-012-2. https://doi.org/10.7476/9786556300122.0021. \section{International license.}

All the contents of this work, except where otherwise noted, is licensed under a Creative Commons Attribution 4.0

Todo o conteúdo deste trabalho, exceto quando houver ressalva, é publicado sob a licença Creative Commons Atribição 4.0. 


\section{Comportamento de industriários quanto à prevenção do câncer da próstata}

André Luis Santos Virgulino Marco Antônio Vasconcelos Rêgo

\section{Introdução}

A saúde do homem vem sendo vista com mais atenção na atualidade. Diversos são os agravos à saúde que possuem grande importância no sexo masculino. Dentre esses, destacam-se os problemas cardiovasculares e os tumores. Entre as neoplasias, o câncer da próstata é um problema de saúde pública de abrangência mundial, e sua incidência anual, padronizada por idade, é de aproximadamente 25,3 casos por 100.000 . (CRAMER, 2007) No Brasil, a incidência é de 52,4 casos por 100.000, ou seja, o dobro da taxa mundial. (INCA, 2005) Em 2008, esta doença foi responsável pela morte de 258.381 homens em todo o mundo. No Brasil, é a segunda causa de morte por câncer entre homens, e desde o ano de 2002 é a primeira na Bahia. (GLOBOCAN, 2008; INCA, 2009)

Parte do aumento na incidência do câncer da próstata é decorrente do aumento da expectativa de vida, e consequentemente do envelhecimento da população, visto que esta neoplasia tem a idade avançada como um dos seus fatores de risco. No Brasil, entre os anos de 1980 e 2005, a expectativa de vida para os homens cresceu de 59,7 para 68,2 anos, ou seja, quase nove anos a mais de vida, decorridos apenas 26 anos. (IBGE, 2009) As neoplasias da próstata são raras antes dos 50 anos e sua incidência aumenta com a idade. Quase $50 \%$ dos indivíduos com 80 anos e, estima-se que, 100\% dos indivíduos que chegaram aos 100 anos têm câncer da próstata, porém, em um número elevado de indivíduos, a doença se apresenta sem sintomas importantes. (SROUGI, 1997) 
A prevenção primária do câncer da próstata é uma tarefa difícil, pois os fatores mais fortemente associados com a doença não são modificáveis, a saber: idade, raça e história familiar. Entre esses, a idade parece ser o mais importante. Outros fatores de risco considerados modificáveis como: vida sedentária; hábitos alimentares; obesidade; tabagismo e alcoolismo também têm sido estudados. (LIU et al., 2000; NORMAN et al., 2002; ROBINSON et al., 2005)

A fase inicial do câncer da próstata é assintomática e já que a prevenção primária ainda não é possível, deve-se trabalhar na prevenção secundária, através da detecção precoce, ou seja, do rastreamento de homens assintomáticos por meio da realização do exame digital retal (EDR) (toque retal como é mais conhecido) e pela dosagem do antígeno prostático específico (PSA). O PSA é uma proteína produzida exclusivamente pela próstata, que se eleva de maneira significativa nos casos de câncer. O EDR é relativamente indolor, ainda insubstituível e presta ao médico importantes informações como: estado do esfíncter anal; estado das fezes dentro do reto; presença de tumores do reto; presença de dor na próstata, vesículas seminais e reto, que podem indicar inflamação; tamanho da próstata; presença de nódulos suspeitos de câncer da próstata; consistência da próstata (dura, mole ou elástica); e estado das bordas, limites e simetria da próstata. (LEFORT; ALMEIDA, 2004) $\mathrm{Na}$ fase mais avançada da doença, os primeiros sintomas são: dificuldade para urinar, jato urinário fraco e aumento do número de micções. (SBU, 2009)

Algumas controvérsias existem sobre a detecção precoce do câncer da próstata.

Embora esses exames possam detectar o câncer da próstata em seus estádios
iniciais, as evidências científicas não permitem concluir se tal detecção reduz
a mortalidade por esse câncer ou melhora a qualidade de vida dos pacientes.
Apesar da detecção e do tratamento precoce prevenirem a progressão do
câncer e o aparecimento de metástases, também é possível que sejam detec-
tados tumores que teriam um crescimento muito lento e que não causariam
problemas à saúde do homem. (INCA, 2005)

Entretanto, admite-se que o diagnóstico precoce aumenta as chances de cura. De $70 \%$ a $98 \%$ dos pacientes são curados quando a doença ainda está alojada dentro da glândula. (SROUGI, 1997) 
Sob a ótica da eficácia, o EDR falha em $30 \%$ a $40 \%$ dos pacientes e o PSA em $20 \%$. O PSA feito em paralelo ao EDR falha em apenas $5 \%$ dos casos, o que justifica a necessidade de realização de ambos os testes. (SROUGI, 1997) Os protocolos internacionais são divergentes sobre os exames e suas combinações para a detecção precoce, porém a maioria inclui o EDR como um dos escolhidos e muitas vezes combinado com a dosagem do PSA. No Brasil, a Sociedade Brasileira de Urologia (SBU) recomenda a realização de exame médico anual, incluindo o EDR, a dosagem sérica do PSA e a dosagem sérica da fração prostática da fosfatase ácida para homens entre 45 e 70 anos de idade, ou a partir dos 40 anos se houver história familiar de câncer da próstata.

Durante séculos, a diferença entre os gêneros foi baseada na relação anatomofisiológica, onde a mulher era vista como o homem invertido. Neste processo, o modelo de perfeição estava representado na anatomia masculina, onde a regra fálica distinguia perfeitamente o domínio de superioridade masculina sobre a feminina. Foi na passagem do século XVIII para o XIX que esta visão mudou e a relação passou a ser a político-ideológica, e as diferenças passaram a ser morais e comportamentais. Neste ponto, a diferença anatômica dos sexos é usada para fundar a diferença de gêneros masculino e feminino, e a partir daí uma série de regras, papéis e traços foram construídos para representar a condição do masculino e do feminino. (ALVES, 2001)

Assim, o masculino é tudo o que se contrapõe ao feminino e para garantir essa soberania, a masculinidade é sempre definida e associada à virilidade, ao poder, à imponência, ao não curvar-se, ao não submeter-se e, assim, seria desonroso para o homem: submeter-se; ser passivo; ficar por baixo; ser impotente; ficar recluso ou restrito no convívio social ou ser invadido. (BOURDIEU, 1999; ALVES, 2004) Porém, levando em consideração o câncer de próstata, estes mesmos aspectos comportamentais e culturais que o enaltecem, são os mesmos que podem contribuir para a ocorrência de desfechos desfavoráveis.

Um dos aspectos que seguramente contribui para a detecção do câncer da próstata é a atitude de muitos homens quanto ao cuidado da saúde como um todo, e no particular quanto aos medos e crenças em torno do EDR. O ambiente de trabalho industrial, por conter normalmente um número maior de homens e atividades que por vezes requerem força física, salienta o comportamento machista que permeia 
a sociedade, e esse comportamento termina por interferir diretamente na relação do trabalhador com a sua saúde.

Dois estudos realizados em ambientes de trabalho encontraram evidências do impacto desse tipo de comportamento no cuidado que os homens dedicam à sua saúde. O primeiro foi realizado com trabalhadores do porto de Santos, em São Paulo, para avaliar a relação do trinômio homem/trabalho/saúde, e reafirmou o modelo ocidental hegemônico de masculinidade, a partir da visão destes trabalhadores sobre o cuidado com a saúde. Para eles, homem é sinônimo de força, virilidade e invulnerabilidade. Nesse contexto, não se permitem estar doentes, e mais, admitem que o cuidado seja algo da natureza feminina. Em seus diálogos, expressam que a saúde é a própria condição de trabalho e a doença é o impedimento para este. A doença os colocaria em um lugar marginal, com risco de perda do trabalho, e consequentemente, da sua condição de provedor. (MACHIN; COUTO; ROSSI, 2009)

O segundo estudo, realizado na Finlândia, avaliou a relação homem, masculinidade e alimentação, comparando engenheiros e carpinteiros. Os resultados apontaram para um comportamento machista mais evidente entre os carpinteiros, justificado pela necessidade de vigor e boa condição física para a realização do seu trabalho, normalmente manual, e diferente do trabalho "leve" dos engenheiros. Os carpinteiros relacionaram o corpo a uma máquina e sua alimentação ao combustível necessário para esta máquina operar. Assim, informaram que enquanto homens necessitam de alimentação pesada, como carne bovina, afirmaram que conhecem os benefícios de uma alimentação mais saudável, rica em vegetais, mas que esta alimentação é coisa de mulher. Os engenheiros também expressaram um consumo de carne, citando o peixe e o frango como alternativas de substituição da carne bovina, além da inclusão de vegetais em suas dietas. (ROOS; PRÄTTÄLÄ; KOSKI, 2001)

Um estudo canadense recente encontrou um menor percentual de homens com idade entre 40 e 60 anos (65\%) quando comparado a mulheres na mesma faixa etária $(79 \%)$ que tenham discutido ou realizado o exame diagnóstico para câncer da próstata ou mamografia, respectivamente. (MCCREARY; GRAY; GRACE, 2006) Não existem no Brasil estudos que façam uma relação entre realização e não realização do EDR como teste de detecção precoce desta neoplasia. Porém, 60\% a 
$70 \%$ dos casos são diagnosticados quando a doença já está disseminada e o tratamento já não é muito eficaz. (SROUGI, 1997)

Muitos são os fatores que poderiam estimular ou inibir um homem a fazer este exame diagnóstico. Alguns estudos realizados no Brasil, Chile, Alemanha e Estados Unidos, já identificaram alguns fatores impeditivos: preocupação quanto ao desconforto físico; embaraço; preocupação com o resultado anormal e crença em algum tipo de distúrbio sexual; falta de tempo, ausência de sintomas urológicos e ausência de dor ou desconforto; falta de informação avaliada em estudos de intervenção; e a masculinidade, sob seus mais variados elementos. (MYERS et al., 1996; NASCIMENTO, 2000; KISS; MERYN, 2001; LUCUMI; CABRERA, 2003; WEIRINCH et al., 2003; KEIJER, 2003; MIRANDA et al., 2004; GOMES, 2008) Como promotores, os já estudados foram: benefício pessoal e presença de sintomas urológicos; educação e informação; e ter histórico familiar. (MYERS et al., 1996; TAYLOR et al., 1999; LEFORT; ALMEIDA, 2004; GOMES; NASCIMENTO; ARAÚJO, 2007)

Muitos outros prováveis fatores impeditivos ou seus desdobramentos ainda não foram bem avaliados, tais como: medo do exame; preconceito; ser muito saudável; descuido pessoal; confiança no resultado do PSA; não ter histórico familiar; falta de acesso à assistência médica; constrangimento com o exame; medo de ficar excitado; desconhecimento da doença; medo da violação anal; custo com o exame; resistência a ir ao médico; achar que cuidados com a saúde é coisa de mulher; vergonha de ficar exposto a outro homem; medo de mudança de orientação sexual; falta de conhecimento do exame; falta de cobertura do plano de saúde; horário do médico inconveniente (mesmo horário do trabalho); não saber que tipo de médico procurar; não saber aonde ir; recusar-se a ir; medo da impotência; ou achar que não precisa do exame. Da mesma forma que os seguintes prováveis promotores: influência de familiares amigos, colegas de trabalho, esposa ou companheiro; solicitação médica; decisão própria; atendimento às campanhas veiculadas em jornais, revistas, televisão, rádio; por consciência da importância do exame para o diagnóstico e saúde; estar na idade de fazer; ou fazer parte de um exame geral de rotina.

No Brasil, há escassez de estudos sobre essa temática, cujos resultados possam indicar a realização de ações voltadas à redução do número de casos diagnosticados em estágios avançados e redução da 
letalidade. O presente estudo visa avaliar o comportamento dos homens quanto à prevenção do câncer de próstata, tomando-se como referência um grupo de trabalhadores da indústria. Essa investigação está em consonância com um dos atuais objetos de atenção do Ministério da Saúde: a saúde do homem. Esse estudo se alinha também a dois dos eixos do Plano de Ação Nacional - PAN (2009), vinculado à Política Nacional de Atenção Integral à Saúde do Homem, qual sejam: a promoção da saúde, informação e comunicação. (BRASIL, 2009a; 2009b)

\section{Notas metodológicas}

Trata-se de um estudo de corte transversal que incluiu 69 trabalhadores com idades entre 42 e 65 anos, pertencentes a três indústrias do Complexo Petroquímico de Camaçari (COPEC). Todos os indivíduos responderam um questionário padronizado e autoaplicável baseado no Modelo de Crenças em Saúde (MCS) de Rosentock, desenvolvido na década de 50. (ROSENTOCK, 1996) Esse modelo prima pela avaliação do comportamento de prevenção em saúde a partir da percepção de cinco dimensões: susceptibilidade; severidade; autoeficácia; benefícios e barreiras para a ação. Além disso, avalia as variáveis demográficas, sociopsicológicas e estruturais.

Como não foram encontrados modelos completos e validados para uso em português, construiu-se um modelo com base em outros três: um sobre prevenção do câncer da próstata; um sobre o câncer da mama; e outro sobre a prevenção de acidentes com agulha. (BREVIDELLI; CIANCIARULLO, 2001; MOORE; BOYLE, 2002; YAN, 2009) O conteúdo das afirmações foi baseado no conhecimento dos pesquisadores e nos fatores identificados nos estudos sobre as barreiras e soluções no comportamento de prevenção encontrado na literatura.

Por se tratar de um instrumento novo, foi realizada uma validação de conteúdo por dois urologistas e uma avaliação de clareza, especificidade e compreensão por dois indivíduos, que atendiam aos critérios de inclusão da pesquisa, mas que não pertenciam ao grupo a ser investigado. Todas as necessidades de revisão identificadas foram reavaliadas. O instrumento final continha 26 questões divididas em quatro partes: características socioeconômicas e culturais; conhecimento sobre 
a doença; características e comportamentos de saúde; e fatores modificadores do comportamento.

As respostas à grande maioria das perguntas foram de escolha simples ou múltipla, dentro de uma lista pré-definida. As respostas aos itens de crença foram obtidas com quatro pontos em escalas tipo Likert (discordo totalmente, discordo, concordo e concordo totalmente). Os valores das escalas do MCS no questionário foram estruturados na direção positiva, ou seja, quanto maior o valor da escala maior a sua percepção.

Os questionários foram entregues aos trabalhadores selecionados pelos médicos do trabalho de suas respectivas empresas. Depois de preenchidos (duração média de 30 minutos), os questionários foram lacrados e guardados em uma pasta. Os dados foram transferidos para uma base de dados sem a identificação dos participantes, havia apenas uma chave de correlação que ficou sobre a guarda dos pesquisadores/ coordenadores. Esta base foi avaliada para certificação da correta transcrição das informações.

Durante a análise, formaram-se dois grupos de indivíduos: trabalhadores que realizaram o EDR e o PSA anualmente (Grupo A) e trabalhadores que só fizeram um ou nenhum deles (Grupo B). Foram realizadas análises descritivas (frequência, média e desvio padrão) para os dados do perfil da população, do conhecimento e do comportamento de saúde. A análise da relação entre as crenças e o comportamento de realização dos exames, foi feita através do cálculo das razões de prevalência.

Para a verificação da validade e confiabilidade das escalas do MCS, utilizou-se o alfa de Cronbach. Para a confirmação de que a escala realmente mede o que se dispõe, foi adotado o critério do $\alpha \geq 0,75$. Desta maneira, a escala severidade para o grupo A e as escalas susceptibilidade, severidade e autoeficácia para o grupo $B$, não demonstraram medir o que se propunha. Assim, a análise foi feita por afirmativa, e não somente pela dimensão. Para a análise das diferenças das médias entre as afirmativas das escalas do modelo, foi utilizado o teste de Mann-Whitney. Utilizou-se o software SPSS (Statistical Package for the Social Sciences) versão 17.0.

O projeto foi aprovado pelo Comitê de Ética em Pesquisa do Instituto de Saúde Coletiva da Universidade Federal da Bahia, através do 
Parecer $n^{\circ} 031 / 2010$. Além disso, todos os participantes da pesquisa assinaram um Termo de Consentimento Livre e Esclarecido, que continha os objetivos do estudo, procedimentos, riscos e proposta de minimização, benefícios, sigilo e privacidade, além da informação de participação voluntária. Os termos de consentimento foram arquivados em uma pasta que foi posteriormente lacrada, de modo a garantir o sigilo das informações.

\section{Resultados}

Os trabalhadores eram na sua maioria brancos, casados, que moram com esposa ou esposa e filhos, católicos, com até o ensino médio de escolaridade e que possuem em média dois filhos. Eles trabalham principalmente em horário administrativo, têm uma média de 22 anos na indústria, com uma renda familiar entre $\mathrm{R} \$ 2.400,00$ e $\mathrm{R} \$ 5.100,00$. Uma comparação entre os homens dos grupos A e B demonstrou uma diferença apenas para a idade, os homens do grupo A tinham idade média de 51,3 anos e os do grupo B, 46,1 anos (Tabela 1).

Tabela 1 - Distribuição dos indivíduos segundo variáveis sociodemográficas e realização de exames para deteç̧ão do câncer da próstata. Salvador, 2011.

\begin{tabular}{l|l|l|l} 
& \multirow{2}{*}{ Características } & \multirow{2}{*}{ Total (\%) } & \multicolumn{2}{c}{ PSA + EDR (anual) } \\
\cline { 3 - 4 } Idade (média em anos) & 48,0 & 51,3 & 46,1 \\
\hline Estado Civil & & & \\
\hline Casado & $53(81,5)$ & $20(83,3)$ & $33(80,5)$ \\
\hline Não casado & $12(18,5)$ & $04(16,7)$ & $08(19,5)$ \\
\hline Escolaridade & & & \\
\hline Até o ensino médio & $42(63,6)$ & $16(66,7)$ & $26(61,9)$ \\
\hline Superior \& Pós-graduação & $24(36,4)$ & $08(33,3)$ & $16(38,1)$ \\
\hline Cor ou Raça & & & \\
\hline Branca & $27(42,2)$ & $07(30,4)$ & $20(48,8)$ \\
\hline Parda & $24(37,5)$ & $08(34,8)$ & $16(39,0)$ \\
\hline Negra & $13(20,3)$ & $08(34,8)$ & $05(12,2)$ \\
\hline Outras & --- & --- & --- \\
\hline Número médio de filhos & 1,9 & 2,2 & 1,78 \\
\hline
\end{tabular}




\begin{tabular}{l|l|l|l}
\hline Com quem mora & & & \\
\hline Esposa \& esposa e filhos & $56(84,8)$ & $22(91,7)$ & $34(81,0)$ \\
\hline Outros & $10(15,2)$ & $02(8,3)$ & $08(19,0)$ \\
\hline Religião ou Culto & & & \\
\hline Católico & $48(70,6)$ & $20(80,0)$ & $28(65,1)$ \\
\hline Não católico & $17(25,0)$ & $05(20,0)$ & $12(27,9)$ \\
\hline Nenhuma/Ateu & $03(4,4)$ & --- & $03(7,0)$ \\
\hline Jornada de Trabalho & & & \\
\hline Administrativo & $56(82,4)$ & $23(92,0)$ & $33(76,7)$ \\
\hline Turno & $12(17,6)$ & $02(8,0)$ & $10(23,3)$ \\
\hline Noturno & --- & --- & --- \\
\hline Renda Familiar & & & \\
\hline$<\mathrm{R} \$ 2.040,00$ & $14(21,2)$ & $05(21,7)$ & $09(21,0)$ \\
\hline $\mathrm{R} \$ 2.041,00-5.100,00$ & $23(34,8)$ & $08(34,8)$ & $15(34,8)$ \\
\hline $\mathrm{R} \$ 5.101,00-10.200,00$ & $19(28,8)$ & $07(30,4)$ & $12(27,9)$ \\
\hline$>\mathrm{R} \$ 10.200,00$ & $10(15,2)$ & $03(13,1)$ & $07(16,3)$ \\
\hline Tempo médio de trabalho na indústria (anos) & 22,0 & 24,6 & 20,7 \\
\hline
\end{tabular}

Fonte: (VIRGULINO; RÊGO, 2011)

\section{Informação em saúde e conhecimento da doença}

Cerca de dois terços dos indivíduos obtém informação ou conselho de como tratar de sua saúde com o médico. Em seguida aparece a esposa ou companheira $(27,9 \%)$. As principais fontes de informação sobre o câncer da próstata foram: jornal ou revista; televisão ou rádio; e médico ou profissional de saúde $(88,4 \%)$. Amigos apareceram em segundo lugar $(75,4 \%)$, seguidos da internet $(65,2 \%)$ e dos familiares $(59,4 \%)$. Dois participantes responderam que não tinham informações sobre esta neoplasia. Como resultado da autoavaliação, 46,2\% dos homens consideraram seu conhecimento como bom e $30,8 \%$ como muito bom ou excelente. Apenas 23,0\% acreditaram ter pouco ou muito pouco conhecimento sobre o câncer da próstata (Tabela 2).

A média geral de acertos obtidos com o questionário de conhecimento foi de $78,6 \%$, e mesmo na comparação entre o Grupo A e B, a diferença entre as médias gerais de acertos é muito pequena, conforme Tabela 2. Os itens deste questionário que apresentaram as menores 
pontuações foram aqueles relativos aos fatores de risco, prevenção e sintomatologia do câncer da próstata. Quanto a esse último aspecto, chamou a atenção, a baixa proporção média de acertos $(21,7 \%)$. No cômputo geral, o grupo A, apresentou frequências médias de acertos mais que $10 \%$ maiores que o grupo $B$. Mesmo com a expressiva proporção de conhecimento demonstrado, 92,2\% dos respondentes gostariam de obter mais informações sobre esta doença.

Tabela 2 - Distribuição dos indivíduos segundo variáveis relacionadas ao conhecimento sobre o câncer da próstata. Salvador, 2011.

\begin{tabular}{l|l|l|l}
\hline \multicolumn{1}{c|}{ Afirmativa } & \multicolumn{3}{|c}{ Acertos (\%) } \\
\cline { 2 - 4 } & \multicolumn{1}{c}{ Total } & \multicolumn{1}{c}{$\begin{array}{c}\text { PSA + EDR } \\
\text { (anual) }\end{array}$} \\
\cline { 2 - 4 } & & Sim & \multicolumn{1}{c}{ Não } \\
\hline $\begin{array}{l}\text { O câncer de próstata é o tipo de câncer mais comum entre os } \\
\text { homens no Brasil. (V) }\end{array}$ & 87,0 & 88,0 & 86,4 \\
\hline $\begin{array}{l}\text { O toque retal é um exame importante no diagnóstico do câncer } \\
\text { de próstata. (V) }\end{array}$ & 98,6 & 100,0 & 97,7 \\
\hline $\begin{array}{l}\text { O “PSA" é um exame de sangue utilizado no diagnóstico do } \\
\text { câncer de próstata. (V) }\end{array}$ & 92,8 & 100,0 & 88,6 \\
\hline $\begin{array}{l}\text { Alguns homens podem ter o câncer de próstata e não ter co- } \\
\text { nhecimento disso. (V) }\end{array}$ & 95,7 & 88,0 & 100,0 \\
\hline $\begin{array}{l}\text { O câncer de próstata pode ser curado se for descoberto (diag- } \\
\text { nosticado) logo no início. (V) }\end{array}$ & 97,1 & 100,0 & 95,5 \\
\hline $\begin{array}{l}\text { O câncer de próstata pode ser prevenido pela realização regular } \\
\text { de exercícios físicos. (F) }\end{array}$ & 68,1 & 76,0 & 63,6 \\
\hline O fumo contribui para o surgimento do câncer de próstata. (F) & 50,7 & 44,0 & 54,5 \\
\hline $\begin{array}{l}\text { A bebida alcoólica contribui para o surgimento do câncer de } \\
\text { próstata. (F) }\end{array}$ & 59,4 & 56,0 & 61,4 \\
\hline $\begin{array}{l}\text { Os homens devem fazer o exame de toque retal anualmente, a } \\
\text { partir dos 60 anos. (F) }\end{array}$ & 73,9 & 84,0 & 68,2 \\
\hline $\begin{array}{l}\text { Os homens devem fazer o exame de PSA anualmente, a partir } \\
\text { dos 45 anos. (V) }\end{array}$ & 82,6 & 88,0 & 79,5 \\
\hline $\begin{array}{l}\text { Os exames da próstata só precisam ser feitos quando aparecem } \\
\text { os sintomas. (F) }\end{array}$ & 97,1 & 100,0 & 95,5 \\
\hline $\begin{array}{l}\text { A forma de descobrir o câncer de próstata antecipadamente é } \\
\text { através de exames. (V) }\end{array}$ & 97,1 & 100,0 & 95,5 \\
\hline $\begin{array}{l}\text { Os principais sintomas do câncer de próstata são jato urinário } \\
\text { fraco e dor nos testículos. (F) }\end{array}$ & 21,7 & 28,0 & 18,2 \\
\hline $\begin{array}{l}\text { A idade é o principal fator de risco para o desenvolvimento do } \\
\text { câncer de próstata. (V) }\end{array}$ & 65,2 & 68,0 & 63,6 \\
\hline
\end{tabular}




\begin{tabular}{l|l|l|l}
\hline Não existe cura para o câncer de próstata. (F) & 91,3 & 88,0 & 93,2 \\
\hline Média & 78,6 & $\mathbf{8 0 , 5}$ & $\mathbf{7 7 , 4}$ \\
\hline \multicolumn{2}{l}{}
\end{tabular}

$\mathrm{V}=$ verdadeiro e $\mathrm{F}=$ falso.

Fonte: (VIRGULINO; RÊGO, 2011)

\section{Percepção, características e comportamento de saúde}

A percepção quanto à própria saúde foi muito positiva, pois $87,3 \%$ dos indivíduos reportaram que consideram sua saúde, de modo geral, muito boa e excelente. Apenas 9,5\% consideraram como boa e 3,2\% como ruim ou muito ruim. Quanto à frequência de visita ao médico, apenas $55,1 \%$ vão anualmente ao clínico, $23,2 \%$ só quando não se sentem bem, $14,5 \%$ a cada dois anos ou mais e $2,9 \%$ só foram uma vez ou não informaram. Apenas 1,4\% dos participantes informaram nunca ter ido ao médico. Se a visita for ao urologista, a frequência é ainda menor. Apenas $42 \%$ reportaram ir anualmente, $15,9 \%$ quando sentem algo, $10,1 \%$ nunca foram ou foram a cada dois anos ou mais e $18,8 \%$ reportaram nunca terem ido ao urologista. A preferência sobre ser o médico urologista do sexo masculino ou feminino está apresentada na Figura 1.

Figura 1 - Distribuição dos indivíduos segundo o sexo do urologista a ser procurado. Salvador, 2011.

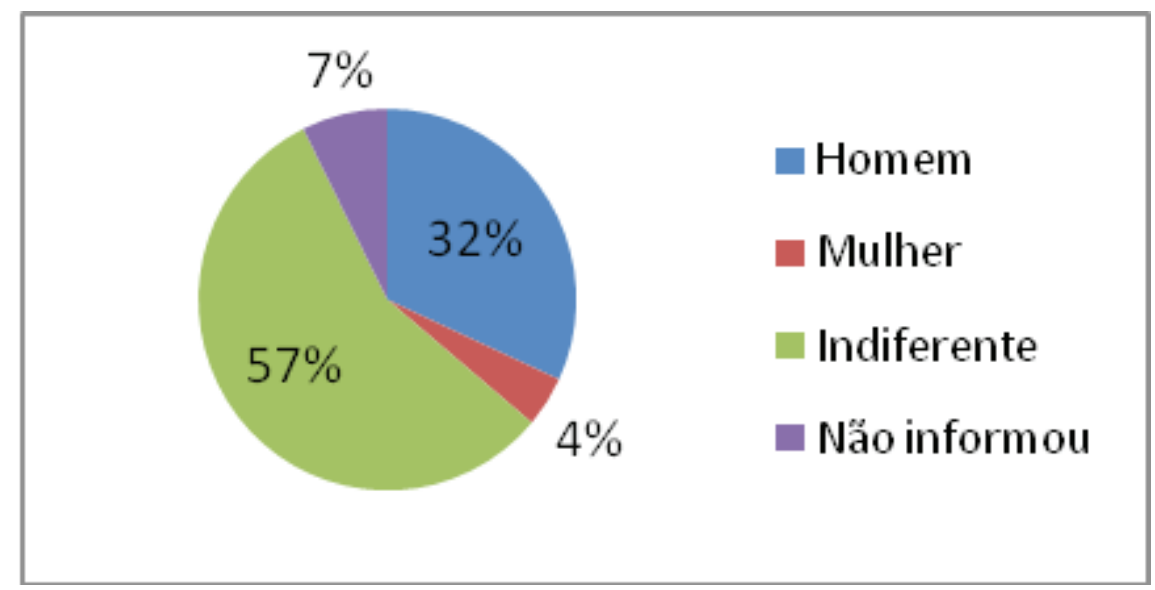

Fonte: (VIRGULINO; RÊGO, 2011) 
Apenas $36 \%$ reportaram ter feito o EDR e dosado o PSA anualmente (Grupo A), contra 64\% que não os fizeram (Grupo B). A Tabela 3 apresenta um resumo dos achados. Uma análise separada dos exames demonstrou que um total de $59,4 \%$ dos participantes realizou o PSA anualmente contra apenas $36,2 \%$ do EDR. De modo geral, a realização do PSA é sempre maior do que a realização do EDR. Muitos participantes $(44,9 \%)$ reportaram nunca terem feito o EDR. Quase um quarto dos participantes relatou histórico familiar de câncer da próstata, com maiores ocorrências entre pai $(14,5 \%)$ e tios $(11,6 \%)$. Uma grande parcela $(46,4 \%)$ reportou desconhecer este histórico familiar (Tabela 4).

Tabela 3 - Distribuição dos indivíduos segundo a frequência de realização dos exames para avaliação da próstata. Salvador, 2011.

\begin{tabular}{l|l|l}
\multicolumn{1}{c|}{ Frequência } & PSA (\%) & EDR (\%) \\
\hline Nunca fez & $11(15,9)$ & $31(44,9)$ \\
\hline Anualmente, por solicitação da empresa & $20(29,0)$ & $08(11,6)$ \\
\hline Anualmente, por iniciativa própria & $21(30,4)$ & $17(24,6)$ \\
\hline A cada dois anos ou mais & $08(11,6)$ & $05(7,2)$ \\
\hline Fez só uma vez & $06(8,7)$ & $07(10,1)$ \\
\hline Desconhece o exame & $01(1,4)$ & -- \\
\hline
\end{tabular}

* O percentual total não reflete \% devido à ausência de resposta de alguns participantes Fonte: (VIRGULINO; RÊGO, 2011)

Tabela 4 - Distribuição dos indivíduos segundo o histórico familiar de câncer próstata. Salvador, 2011.

\begin{tabular}{l|c|c}
\multicolumn{1}{c|}{ Grau de parentesco } & N & $\% *$ \\
\hline Desconhece & 32 & 46,4 \\
\hline Não existe histórico & 24 & 34,8 \\
\hline Pai & 10 & 14,5 \\
\hline Tio & 08 & 11,6 \\
\hline Avô & 03 & 4,3 \\
\hline Primo & -- & -- \\
\hline Irmão & -- & -- \\
\hline
\end{tabular}

* O percentual total não reflete \% devido a múltiplas respostas dos participantes.

Fonte: (VIRGULINO; RÊGO, 2011) 


\section{Análise da influência das crenças sobre o comportamento de prevenção}

Segundo o MCS, o comportamento de prevenção de uma doença pode ser explicado através da relação existente entre a percepção de cinco dimensões e a possibilidade da ação preventiva. Estas dimensões são: susceptibilidade; severidade; autoeficácia; benefícios e barreiras. Fazendo uma análise geral da relação entre o grau de percepção das dimensões do MSC e sua influência na realização do rastreamento anual, foi observado que uma percepção mais alta das dimensões susceptibilidade, severidade, autoeficácia e benefício contribuíram para uma maior realização dos exames anuais. Contraditoriamente, a percepção mais alta de barreiras também proporcionou uma maior frequência de realização dos exames (Tabela 5). Uma análise mais detalhada do MCS foi realizada comparando os resultados das médias encontradas de cada dimensão do modelo entre os Grupos A e B. Todos os resultados sumarizados nas Tabelas 6 e 7 .

\section{Percepção de susceptibilidade}

A susceptibilidade percebida refere-se à avaliação individual de chances de adquirir uma doença ou de vivenciá-la. Os resultados encontrados demonstram não haver diferença significativa entre as médias encontradas para os dois grupos comparados $(p=0,258)$. Mesmo na avaliação de cada uma das cinco afirmativas que compõem esta dimensão, não foi encontrada diferença significativa ( $p>0,05)$. Assim, conclui-se que os dois grupos analisados apresentam uma mesma percepção de susceptibilidade e que esta dimensão não influiu no comportamento de prevenção do câncer da próstata para a população estudada.

\section{Percepção de severidade}

A severidade percebida é o julgamento individual do quão severo é a doença ou seus desdobramentos. A percepção geral de severidade entre os dois grupos não apresentou diferença significativa, porém na avaliação individual de cada uma das nove afirmativas que compõe esta dimensão, uma apresentou esta diferença. A afirmativa "o tratamento deste câncer causa impotência” foi maior em 21,4\% para o grupo B. 
Desta forma, essa circunstância parece ter influenciado de modo inverso, ou seja, ao invés de atuar na percepção de severidade se tornou uma barreira para a realização dos exames.

De modo geral, assim como a susceptibilidade, a percepção de severidade foi igual entre os dois grupos, e não atuou na motivação de realização dos exames da próstata.

\section{Percepção de autoeficácia}

A percepção de autoeficácia refere-se à crença pessoal na própria capacidade de fazer algo, neste caso, do cuidado com a própria saúde. Observando os resultados gerais, foi verificada uma diferença de 16,7\% maior da percepção de autoeficácia do grupo A $(p=0,021)$. A análise individual apontou para a diferença na afirmativa "eu me sinto saudável tomando conta da minha própria saúde”. Assim, verificou-se que esta percepção influenciou no comportamento de prevenção, motivando os trabalhadores a realizar os exames para diagnóstico precoce.

\section{Percepção de benefícios}

A percepção de benefícios é uma conclusão individual de que uma nova ação lhe trará resultados positivos. Uma ação pode ser vista como benéfica se esta atua na redução da susceptibilidade ou na severidade, ou em ambas, da doença. Fazendo a comparação geral da percepção de benefícios entre os dois grupos, não foi encontrada diferença significativa entre suas médias $(p=0,351)$. A mesma conclusão foi feita na análise individual de cada afirmativa que compõe esta dimensão. Desta forma, a percepção de benefícios também não implicou em um maior comportamento de realização do rastreamento anual.

\section{Percepção de barreiras}

A percepção de barreiras é uma visão individual de todos os aspectos negativos da ação em saúde que interferem na prevenção. Das dimensões do MCS avaliadas, e que apresentaram diferenças significativas entre suas médias, a percepção de barreiras foi a que resultou em um maior valor $(28,8 \%)$. Sua influência foi no comportamento não 
preventivo, ou seja, as barreiras percebidas inibiram os trabalhadores a realizarem os exames de prevenção.

$\mathrm{Na}$ avaliação detalhada, 13 afirmativas apresentaram diferenças significativas entre suas médias na comparação entre os grupos A e B, variando de $21,9 \%$ a $45,3 \%$. Da mesma forma que a média geral da dimensão, estas 13 afirmativas atuaram negativamente no comportamento de prevenção do câncer. Agrupando-se essas 13 afirmativas, observam-se três aspectos que atuaram como barreira para a realização dos exames de detecção precoce do câncer da próstata.

O primeiro deles foi a masculinidade, composto pelas afirmativas: "eu tenho medo de descobrir que estou com câncer da próstata"; "eu tenho medo de fazer o EDR"; "eu não faço os exames da próstata porque me sinto uma pessoa muito saudável"; "eu não faço os exames da próstata porque sou descuidado com minha saúde"; "eu não faço os exames da próstata porque não gosto de ir ao médico"; "eu tenho medo de ter ereção durante o EDR"; e "eu tenho medo de mudar de orientação sexual ao fazer o EDR".

Em segundo lugar, a falta de conhecimento sobre a doença, com quatro barreiras identificadas, composta pelas seguintes afirmativas: "eu tenho medo de sentir dor durante o EDR"; "eu não faço os exames porque não tenho sintomas"; "eu não conheço quais são os métodos de prevenção do câncer da próstata"; e "eu não sei que médico devo procurar para fazer os exames da próstata". Por último, com duas barreiras identificadas, a barreira médica, composta pelas afirmativas: "o médico me informou que não é necessário fazer os exames da próstata" e "nenhum médico me solicitou os exames da próstata". Os valores e as diferenças entre as médias obtidas na dimensão e em cada afirmativa podem ser encontrados na Tabela 5 . 
Tabela 5 - Distribuição dos indivíduos, segundo as dimensões do Modelo de Crenças em Saúde e situação de rastreamento. Salvador, 2011.

\begin{tabular}{|c|c|c|c|c|}
\hline \multirow{3}{*}{ Dimensão/Afirmativas } & \multirow{2}{*}{\multicolumn{2}{|c|}{$\begin{array}{c}\text { Pontuação média } \\
\begin{array}{c}\text { EDR + PSA } \\
\text { anual }\end{array}\end{array}$}} & \multirow{3}{*}{$\Delta \%$} & \multirow{3}{*}{$\begin{array}{c}\text { p- } \\
\text { valor* }\end{array}$} \\
\hline & & & & \\
\hline & Sim & Não & & \\
\hline Susceptibilidade percebida & 2,65 & 2,48 & 6,9 & 0,258 \\
\hline O câncer de próstata é uma doença inevitável. & 2,09 & 2,11 & $-0,9$ & 0,541 \\
\hline Eu fico preocupado em um dia ter câncer de próstata. & 3,00 & 2,65 & 13,2 & 0,127 \\
\hline $\begin{array}{l}\text { Eu tenho chances de desenvolver o câncer de } \\
\text { próstata. }\end{array}$ & 2,73 & 2,78 & $-1,8$ & 0,571 \\
\hline É provável que eu venha a ter câncer de próstata. & 2,50 & 2,27 & 10,1 & 0,269 \\
\hline $\begin{array}{l}\text { Com o passar dos anos eu tenho mais chances de } \\
\text { ter CaP. }\end{array}$ & 2,91 & 2,59 & 12,4 & 0,199 \\
\hline Severidade percebida & 2,87 & 2,87 & 0,0 & 0,817 \\
\hline O câncer de próstata é uma doença grave. & 3,55 & 3,29 & 7,9 & 0,078 \\
\hline $\begin{array}{l}\text { O câncer de próstata resulta em morte se não for } \\
\text { tratado. }\end{array}$ & 3,73 & 3,68 & 1,4 & 0,880 \\
\hline O tratamento deste câncer causa impotência. & 2,50 & 3,18 & $-21,4$ & 0,004 \\
\hline Eu seria um peso para a minha família se tivesse CaP. & 2,50 & 2,41 & 3,7 & 0,747 \\
\hline $\begin{array}{l}\text { Ter CaP poderia afetar a minha vida social e } \\
\text { profissional. }\end{array}$ & 2,91 & 2,65 & 9,8 & 0,160 \\
\hline Assusta-me pensar que um dia eu possa ter CaP. & 2,95 & 3,06 & $-3,6$ & 0,734 \\
\hline Eu ficaria com baixa autoestima se tivesse CaP. & 2,95 & 3,00 & $-1,7$ & 0,818 \\
\hline $\begin{array}{l}\text { Minha segurança financeira estaria ameaçada se eu } \\
\text { tivesse CaP. }\end{array}$ & 2,59 & 2,59 & 0,0 & 0,870 \\
\hline Não existe cura para o câncer de próstata. & 2,18 & 1,94 & 12,4 & 0,299 \\
\hline Autoeficácia & 2,86 & 2,45 & 16,7 & 0,021 \\
\hline
\end{tabular}




\begin{tabular}{|c|c|c|c|c|}
\hline $\begin{array}{l}\text { Eu me sinto saudável tomando conta da minha } \\
\text { própria saúde. }\end{array}$ & 2,78 & 1,95 & 42,6 & 0,001 \\
\hline Eu sinto que tenho o controle do meu corpo. & 2,65 & 2,62 & 1,1 & 0,781 \\
\hline Eu sou responsável pela minha saúde. & 3,13 & 2,79 & 12,2 & 0,075 \\
\hline Benefício percebido & 3,38 & 3,27 & 3,4 & 0,351 \\
\hline $\begin{array}{l}\text { O EDR ajuda na detecção precoce do câncer de } \\
\text { próstata. }\end{array}$ & 3,61 & 3,21 & 12,5 & 0,201 \\
\hline $\begin{array}{l}\text { Se o câncer for detectado precocemente, o tempo de } \\
\text { tratamento será menor. }\end{array}$ & 3,39 & 3,50 & $-3,1$ & 0,307 \\
\hline O exame de PSA ajuda na detecção precoce do $\mathrm{CaP}$. & 3,48 & 3,39 & 2,7 & 0,846 \\
\hline Eu acredito que o exame de PSA é confiável. & 3,26 & 3,05 & 6,9 & 0,394 \\
\hline $\begin{array}{l}\text { Seguindo as recomendações médicas, eu cuido } \\
\text { melhor da minha saúde. }\end{array}$ & 3,39 & 3,29 & 3,0 & 0,625 \\
\hline $\begin{array}{l}\text { Eu acredito que o EDR é um procedimento de fácil } \\
\text { realização. }\end{array}$ & 3,17 & 3,11 & 1,9 & 0,803 \\
\hline $\begin{array}{l}\text { O CaP pode ser curado se for descoberto logo no } \\
\text { início. }\end{array}$ & 3,39 & 3,26 & 4,0 & 0,515 \\
\hline Eu acredito que o exame de toque retal é confiável. & 3,35 & 3,32 & 0,9 & 0,852 \\
\hline $\begin{array}{l}\text { Eu acredito que o exame de PSA é um procedimento } \\
\text { de fácil realização. }\end{array}$ & 3,39 & 3,29 & 3,0 & 0,645 \\
\hline
\end{tabular}

* Teste de Mann-Whitney.

Fonte: (VIRGULINO; RÊGO, 2011)

Tabela 6 - Distribuição dos indivíduos, segundo a situação de rastreamento e os constructos do modelo de crenças em saúde. Salvador, 2011.

\begin{tabular}{|c|c|c|c|}
\hline \multirow[t]{2}{*}{ Dimensão } & \multicolumn{2}{|c|}{$\begin{array}{c}\text { (\%) Informado } \\
\text { EDR + PSA anual }\end{array}$} & \multirow{2}{*}{$\begin{array}{c}\text { Razão de } \\
\text { Prevalência }\end{array}$} \\
\hline & $\operatorname{Sim}(\%)$ & Não (\%) & \\
\hline Susceptibilidade & & & 1,58 \\
\hline Alta - média & $12(52,2)$ & $15(34,9)$ & \\
\hline Baixa - nenhuma & $11(47,8)$ & $28(65,1)$ & \\
\hline Severidade & & & 1,54 \\
\hline Alta - média & $20(87,0)$ & $32(78,0)$ & \\
\hline Baixa - nenhuma & $03(13,0)$ & $09(22,0)$ & \\
\hline
\end{tabular}




\begin{tabular}{l|c|c|c}
\hline Autoeficácia & & & 1,71 \\
\hline Alta - média & $16(69,6)$ & $20(50,0)$ & \\
\hline Baixa - nenhuma & $07(30,4)$ & $20(50,0)$ & \\
\hline Benefícios & & & 1,08 \\
\hline Alta - média & $22(95,7)$ & $39(95,1)$ & \\
\hline Baixa - nenhuma & $01(4,3)$ & $02(4,9)$ & \\
\hline Barreiras & & & 1,18 \\
\hline Alta - média & $03(16,7)$ & $05(13,5)$ & \\
\hline Baixa - nenhuma & $15(83,3)$ & $32(86,5)$ & \\
\hline
\end{tabular}

Alta - média $=$ respostas concordo e concordo totalmente.

Baixa - nenhuma $=$ respostas discordo e discordo totalmente.

Fonte: (VIRGULINO; RÊGO, 2011)

Tabela 7 - Distribuição dos indivíduos, segundo a consistência dos valores das dimensões do Modelo de Crenças em Saúde por situação de rastreamento. Salvador, 2011.

\begin{tabular}{|c|c|c|c|c|}
\hline \multirow{3}{*}{ Dimensão } & \multicolumn{4}{|c|}{ EDR + PSA annual } \\
\hline & \multicolumn{2}{|c|}{ Sim } & \multicolumn{2}{|c|}{ Não } \\
\hline & Média & $\begin{array}{c}\text { Alfa de } \\
\text { Cronbach }\end{array}$ & Média & $\begin{array}{l}\text { Alfa de } \\
\text { Cronbach }\end{array}$ \\
\hline Susceptibilidade & 2,65 & 0,752 & 2,48 & 0,167 \\
\hline Severidade & 2,87 & 0,660 & 2,87 & 0,676 \\
\hline Autoeficácia & 2,86 & 0,777 & 2,45 & 0,408 \\
\hline Benefícios & 3,38 & 0,962 & 3,27 & 0,857 \\
\hline Barreiras & 1,52 & 0,958 & 2,12 & 0,915 \\
\hline
\end{tabular}

Fonte: (VIRGULINO; RÊGO, 2011)

\section{Análise da influência das características sociodemográficas sobre o comportamento de prevenção}

Observou-se que idade, estado civil, escolaridade, religião e tempo de trabalho demonstraram ter alguma influência positiva no comportamento de prevenção. Os demais fatores analisados, como raça, número de filhos, renda mensal, histórico familiar e grau de conhecimento 
testado não apresentaram nenhuma influência neste comportamento (Tabela 8).

Tabela 8 - Razões de prevalência segundo variáveis soiodemográficas e situação de rastreamento. Salvador, 2011.

\begin{tabular}{|c|c|c|c|}
\hline \multirow{3}{*}{ Variável } & \multirow{2}{*}{\multicolumn{2}{|c|}{$\begin{array}{c}\text { (\%) Informado } \\
\text { EDR + PSA anual }\end{array}$}} & \multirow{3}{*}{$\begin{array}{c}\text { Razão de } \\
\text { Prevalência }\end{array}$} \\
\hline & & & \\
\hline & $\operatorname{Sim}(\%)$ & Não (\%) & \\
\hline Total de participantes & 24 & 41 & \\
\hline Idade (anos) & & & 3,12 \\
\hline $50-59$ & $17(70,8)$ & $11(27,5)$ & \\
\hline $40-49$ & $07(29,2)$ & $29(72,5)$ & \\
\hline Estado civil & & & 1,21 \\
\hline Casado & $20(80,0)$ & $33(75,0)$ & \\
\hline Não casado & $05(20,0)$ & $11(25,0)$ & \\
\hline Grau de escolaridade & & & 1,14 \\
\hline Menor ou igual ao ensino médio & $16(66,7)$ & $26(61,9)$ & \\
\hline Maior que o ensino médio & $08(33,3)$ & $16(38,1)$ & \\
\hline Cor ou raça & & & 0,60 \\
\hline Branca & $07(30,4)$ & $20(48,8)$ & \\
\hline Não branco & $16(69,6)$ & $21(51,2)$ & \\
\hline Número de filhos & & & 0,56 \\
\hline$\leq 02$ & $15(62,5)$ & $33(82,5)$ & \\
\hline$>02$ & $09(37,5)$ & $07(17,5)$ & \\
\hline Religião & & & 1,67 \\
\hline Católico & $20(80,0)$ & $28(65,1)$ & \\
\hline Não católico & $05(20,0)$ & $15(34,9)$ & \\
\hline Renda mensal & & & 1,02 \\
\hline$\leq \mathrm{R} \$ 5.100,00$ & $13(56,5)$ & $24(55,8)$ & \\
\hline$>\mathrm{R} \$ 5.100,00$ & $10(43,5)$ & $19(44,2)$ & \\
\hline Tempo de trabalho (anos) & & & 2,16 \\
\hline$>20$ & $16(76,2)$ & $21(51,2)$ & \\
\hline$\leq 20$ & $05(23,8)$ & $20(48,8)$ & \\
\hline Histórico familiar de câncer da próstata & & & 0,41 \\
\hline Sim & $02(10,0)$ & $07(31,8)$ & \\
\hline Não & $18(90,0)$ & $15(68,2)$ & \\
\hline Grau de conhecimento testado & & & 0,62 \\
\hline
\end{tabular}




\begin{tabular}{l|l|l|l}
\hline Muito pouco - muito bom & $13(52,0)$ & $31(70,5)$ & \\
\hline Excelente & $12(48,0)$ & $13(29,5)$ & \\
\hline
\end{tabular}

Fonte: (VIRGULINO; RÊGO, 2011)

\section{Discussão}

O objetivo deste estudo foi avaliar o comportamento de prevenção do câncer da próstata entre industriários. Os resultados demonstram que a população estudada não frequenta muito os consultórios médicos e a taxa de realização dos exames da próstata é muito pequena. Não foram encontrados estudos que investigassem a frequência de realização entre trabalhadores, mas normalmente elas não são muito altas, com uma média de 40\%. (TALAVERA et al., 2002; CORMIER et al., 2003)

O conhecimento sobre o câncer da próstata apresentou bons resultados. Outros estudos apresentaram níveis de conhecimento semelhantes, corroborando com o resultado encontrado. (CORMIER et al., 2003) Porém, quando se analisaram as barreiras percebidas no MCS constatou-se que este conhecimento não foi forte o suficiente para promover as ações de detecção precoce, uma vez que algumas barreiras foram relacionas à falta ou pouco conhecimento em algum item específico.

A análise do MCS demonstrou que o aumento nas percepções de susceptibilidade, severidade, autoeficácia e benefício contribuem com a prevenção do câncer da próstata, através do aumento da frequência de realização dos exames. Este é o comportamento esperado destas dimensões neste modelo e encontrado em outros estudos que usaram esta metodologia. (BREVIDELLI; CIANCIARULLO, 2001) A contradição surgiu apenas na dimensão das barreiras, pois a regra é que a maior prevenção ocorra no grupo com menor percepção. Este fato pode ser explicado pelo tamanho da amostra, que talvez não tenha sido grande o suficiente para representar melhor esta dimensão.

A avaliação das escalas e de suas afirmativas entre os grupos A e B não apresentaram diferenças significativas nas dimensões susceptibilidade, severidade e benefícios percebidos. Isso se traduz na concepção de que os dois grupos possuem a mesma percepção destas dimensões e que elas não influenciaram no comportamento de prevenção. Uma afir- 
mativa surgiu na dimensão severidade de modo negativo. A afirmativa "o tratamento deste câncer causa impotência" foi a única a apresentar diferença significativa entre os dois grupos, porém com uma percepção maior do Grupo B, o que pode ser traduzido como mais uma barreira. $\mathrm{O}$ medo da possibilidade de perda da virilidade, característica marcante do comportamento machista, aparenta atuar como uma barreira neste caso.

A dimensão autoeficácia, como esperado, apresentou diferença significativa entre os grupos, sendo maior no grupo A. A afirmativa deste grupo de maior expressão foi "eu me sinto saudável tomando conta da minha própria saúde”. Uma contradição do modelo machista hegemônico, no qual o comportamento de cuidado com a saúde é de responsabilidade da mulher. Este pensamento da responsabilidade feminina do cuidado com a saúde foi identificado como uma barreira na prevenção do câncer da próstata no estudo de Madjar, Denham e Rashid (2007). Assim, esta percepção de autoeficácia aparece como uma solução para a prevenção.

A percepção das barreiras foi a mais marcante. Apesar da primeira análise do MCS ter indicado um maior comportamento de prevenção entre os homens com a percepção de barreiras mais altas, nesta análise mais detalhada isto é retificado. A diferença entre as médias de cada grupo foi significativa para o grupo B. Isso indica que quanto maior a percepção das barreiras, menor será o comportamento preventivo, premissa essa esperada do modelo e encontrada em alguns estudos. (LUCUMÍ; CABRERA, 2005)

As barreiras com diferença significativa entre os grupos foram organizadas em três categorias: masculinidade; falta de conhecimento; e barreiras médicas. A masculinidade, ou o comportamento machista e sua relação com o cuidado da própria saúde parece ser o elemento mais estudado quando se trata do assunto prevenção do câncer da próstata. Diversos autores, como Lucumí e Cabrera (2005), Bloom e colaboradores (2006) e Gomes e colaboradores (2008) pesquisaram e elucidaram esta barreira como a mais evidente. Neste estudo, foi esta categoria que apresentou maior número de barreiras e com maior intensidade. Uma parcela dos trabalhadores que não fizeram o exame acredita que: o EDR dói durante a sua realização, o que não ocorre se for feito por um profissional experiente e treinado; diz não fazer o exame por falta de sintomas, quando é sabido que esta neoplasia é assintomática no início; 
informa não conhecer os métodos de prevenção ou qual especialidade médica procurar.

Estas mesmas barreiras relacionadas à falta de conhecimento foram encontradas por Reynolds (2008) e Patel e colaboradoes (2010) em seus estudos realizados em Afro-Americanos. Isto sugere a necessidade de promoção na educação sobre a próstata com ênfase na sintomatologia, fatores de risco e exames de prevenção. Por sua vez, Paiva, Motta e Griep (2010) encontraram no seu estudo uma prevalência de 7,6 $(\mathrm{IC} 95 \%=2,4-23,6)$ para referência de prática adequada dos exames, entre homens que apresentaram conhecimento adequado do que a encontrada entre aqueles que demonstraram conhecimento inadequado.

Por fim, as barreiras médicas. Estas são encontradas na literatura como sendo relativas à pouca interação médico/paciente; não solicitação dos exames por parte do médico, que pode ser uma representação da falta de um protocolo de rastreamento instituído; e não confiar ou gostar de ir ao médico. (ROBINSON; ASHLEY; HAYNES, 1996; REYNOLDS, 2008;) A primeira observação que se pode fazer sobre esta categoria é a baixa frequência relatada de visita ao médico clínico, anualmente, e ainda menor ao urologista. Segundo, uma das afirmativas que foi categorizada como masculinidade também se adapta aqui. No MCS a afirmativa "eu não faço os exames da próstata porque não gosto de ir ao médico" é maior no grupo que não faz os exames anualmente. Em seguida, as afirmativas "o médico me informou que não é necessário fazer os exames" e "nenhum médico me solicitou ainda", também são mais evidentes no grupo B, pois reforçam uma falta de atuação ou atenção médica a este problema de saúde pública.

Alguns achados apontam barreiras relacionadas ao acesso à assistência médica. (PATEL et al., 2010; ROBINSON; ASHLEY; HAYNES, 1996) Entretanto, este item não foi avaliado porque a população estudada dispõe de médico do trabalho na empresa e de assistência médica particular.

\section{Limitações do estudo}

O estudo apresentou quatro limitações. A primeira refere-se ao caráter não-probabilístico da amostragem. Porém, os achados podem 
ser aplicados às situações onde a população apresente as mesmas características deste estudo.

A segunda limitação está relacionada ao fato de que as informações sobre realização dos exames (PSA e EDR) foram autoreportadas, o que pode superestimar o resultado encontrado, diante da tendência dos indivíduos a dar as respostas que satisfaçam ao pesquisador. Com isso, a frequência de realização do PSA e do EDR pode ser menor do que as descritas.

A terceira surgiu na comparação do comportamento de prevenção entre os grupos A e B. Para esta comparação, seriam necessários 52 participantes para cada grupo. A amostra foi composta por 24 (46,2\%) participantes do grupo A e $45(86,5 \%)$ do grupo B, e isso reduziu o poder do estudo. Esta limitação foi criada pela dificuldade que ocorreu durante a coleta dos dados em função da não devolução de alguns questionários. Por fim, assumindo que os componentes do grupo A não apresentariam barreiras para o comportamento de prevenção, eles não precisariam responder a esta pergunta. Assim, apenas 14 participantes dos 24 que compunham o grupo responderam. Mesmo assim, realizouse uma comparação entre grupos.

\section{Considerações finais}

Este é o primeiro estudo no Brasil a utilizar o MCS e a estudar os trabalhadores da indústria para a análise do comportamento de prevenção do câncer da próstata. Seus resultados podem, até certo ponto, contribuir na orientação de estratégias locais para a prevenção desta neoplasia.

Neste modelo, variáveis como idade, situação socioeconômica, educação e conhecimento atuam diretamente nas percepções de susceptibilidade, severidade, autoeficácia e benefícios. Estas atuam sobre as barreiras percebidas, minimizando-as, influenciando no comportamento de prevenção a ser adotado. Dentre estas variáveis, as mais evidentes no comportamento de prevenção foram masculinidade e conhecimento. Porém, a partir da percepção das respostas encontradas, admite-se que um melhor conhecimento pode atuar sobre o comportamento machista desta população de modo a reduzi-lo, permitindo direcionar estes trabalhadores para a prática preventiva. 
Este conhecimento poderia advir de um Programa de Educação e Comunicação em Saúde da Próstata, com o objetivo de maximizar o conhecimento atual e esclarecer as dúvidas encontradas. Este programa deve ter um foco na sintomatologia, exames de detecção, fatores de risco e tratamento desta neoplasia. Além disso, deve ser levada em consideração a inclusão da participação das mulheres/companheiras. Apesar de não ter surgido como uma barreira significativa, elas foram, com $27,9 \%$, a segunda pessoa a quem os pesquisados mais procuraram para conselhos de como tratar de sua saúde, atrás apenas para os médicos.

\section{Referências}

ADDIS, M. E.; MAHALIK, J. R. Men, masculinity and the contexts of help seeking. American Psychologist Association, v. 58, n. 1, p. 5-14, 2003.

ALMEIDA, J. A implantação da indústria automobilística no Brasil. Rio de Janeiro: FGV, 1972.

ALVES, J. E. D. O discurso da dominação masculina. In: GENERAL Population Conference IUSSP International Union For The Scientific Study of Population, 24. Salvador, 2001. . A linguagem e as representações da masculinidade. Rio de Janeiro: Escola Nacional de Ciências Estatísticas, 2004. 33p. BLOOM, J. R. et al. Family history, perceived risk, and prostate cancer screening among African American men. Cancer Epidemiol Biomarkers Prev, n. 15, p. 2167-2173. 2006. BOURDIEU, P. A dominação masculina. Rio de Janeiro: Bertrand Brasil, 1999.

BRASIL. Ministério da Saúde. Sistema de Informação sobre Mortalidade/DATASUS, 2010. Disponível em: <http:// www. datasus. gov. br. $>$ Acesso em: 18 out. 2010. . Ministério da Saúde. Secretaria de Atenção à Saúde, Departamento de Ações Programáticas e Estratégicas Política nacional de atenção integral à saúde do homem: princípios e diretrizes. Brasília: Ministério da Saúde 2009a. . Ministério da saúde, Secretaria de atenção à Saúde, Departamento de Ações Programáticas Estratégicas. Plano de ação 
nacional 2009-2011 da Política nacional de atenção integral á saúde do homem. Brasília: Ministério da Saúde, 2009b.

BREVIDELLI, M. M.; CIANCIARULLO, T. I. Aplicação do modelo de crenças em saúde na prevenção dos acidentes com agulha. Revista de Saúde Pública, São Paulo, v. 35, n. 2, abr. 2001.

CORMIER, L. et al. Screening behavior in brothers and sons of men with prostate cancer. J. Urol. , v. 169, n. 5 p. 1715-1719. May. 2003.

CRAMER, S. D. Prostate cancer: deadly diseases and epidemics. [S. 1]: Chealsea House, 2007. 112p.

GLOBOCAN 2008 (IARC), Section of Cancer Information. Disponível em: < http://globocan. iarc. fr/>. Acesso em: 27 abr. 2010.

GOMES, R. Sexualidade masculina, gênero e saúde. 1. ed. Rio de Janeiro: Fiocruz, 2008.

GOMES, R. et al. As Arranhaduras da masculinidade: uma discussão do toque retal como medida de prevenção do câncer prostático. Ciência e Saúde Coletiva, Rio de Janeiro, v. 13, n. 6, nov. /dez. , 2008.

; NASCIMENTO, E. F.; ARAÚJO, F. C. Por que os homens

buscam menos os serviços de saúde do que as mulheres? As explicações de homens com baixa escolaridade e homens com ensino superior. Cadernos de Saúde Pública, v. 23, p. 565-574, 2007.

IBGE - Instituto Brasileiro de Geografia e Estatística. Tábuas de vidas. Disponível em: <www. ibge. gov. br>. Acesso em: 26 out. 2009.

INCA - Instituto Nacional de Câncer. Síntese de resultados e comentários, 2005. Disponível em: <http://www. inca. gov. br/estimativa/2008 >. Acesso em: 15 abr. 2008.

. Instituto Nacional de Câncer. Hábitos alimentares, 2009.

Disponível em: < http://www. inca. gov. br/>. Acesso em: 20 out. 2009.

KEIJZER, B. Hasta donde el cuerpo aguante: género, cuerpo y salud masculina. In: CÁCERES, C. F et al. La salud como derecho ciudadano: perspectivas y propuestas desde América Latina. Lima: Universidad Peruana Cayetano Heredia; 2003. p. 137-152.

KISS, A.; MERYN, S. Effect of sex and gender on psychosocial aspects of prostate and breast cancer. BMJ, v. 323, Nov, 2001.

LEFORT, A; ALMEIDA, J. C. Câncer de próstata: a importância das campanhas de prevenção. Belo Horizonte: CIAPE, 2004. 
LIU, S. et al. A prospective study of physical activity and risk of prostate cancer in US physicians. International Journal of Epidemiology. n. 29, p. 29-35, 2000.

LUCUMÍ, D.; CABRERA, G. Creencias sobre examen digital rectal como tamiz para cáncer en la próstata: hallazgos cualitativos de un estudio en Cali. Colomb Med, n. 34, p. 112-119. 2003.

. Beliefs of men from Cali, Colombia, about digital rectal examination: findings of an exploratory study. Caderno de Saúde Pública, Rio de Janeiro, v. 21, n. 5, Out. 2005.

MACHIN, R; COUTO, M. T.; ROSSI, C. C. S. Representações de trabalhadores portuários de Santos-SP sobre a relação trabalhosaúde. Saúde soc. , São Paulo, v. 18, n. 4, dez. 2009.

MADJAR, I.; DENHAM, J.; RASHID, P. Do women have a role in early detection of prostate cancer? Lessons from a qualitative study. Australian Family Physician, v. 36, n. 5, May 2007.

MCCREADY, D. R.; GRAY, R. E.; GRACE, S. L. Gender differences in cancer mortality risk perceptions and screening behaviors among adults 40-60 tears of age. International Journal of Men's Health, v. 5, n. 1, p. 53-63.

MIRANDA, P. S. C. et al. Práticas de diagnóstico precoce de câncer de próstata entre professores da faculdade de medicina - UFMG.

Rev. Assoc. Med. Bras., São Paulo, v. 50, n. 3, 2004.

MOORE, A. D. M.; BOYLE, G. Assessing the knowledge, self-efficacy and health behaviors of male beneficiaries assigned to the national capital area regarding participation in prostate screening. 2002. Dissertação (Mestrado). Uniformed Services, University of the Health Sciences, 2002.

MYERS, R. E. et al. Factors associated with intention to undergo annual prostate cancer screening among African American men in Philadelphia. American Cancer Society. v. 78, n. 3, p. 471-479, Aug. 1996.

NASCIMENTO, M. R. Câncer de próstata e masculinidade: motivações e barreiras para a realização do diagnóstico precoce da doença. 2000. Disponível em < http://www. abesp. nepo. unicamp. br. > Acesso em: 20 maio 2010.

NIJS, H. G. T. et al. Why do men refuse or attend population-based screening for prostate cancer? Faculty of Public Health Medicine, v. 22, n. 3, p. 312-316, 2000.

NORMAN, A. et. al. Occupational physical activity and risk for prostate cancer in a nationwide cohort study in Sweden. Br J Cancer. v. 86, n. 1, p. 70-75, Jan. 2002. 
PAIVA, E. P.; MOTTA, M. C. S.; GRIEP, R. H. Conhecimentos, atitudes e práticas acerca da detecção do câncer de próstata. Acta Paul Enferm, v. 23, n. 1, p. 88-93. 2010.

PATEL, K. et al. Factors influencing prostate cancer screening in lowincome African Americans in Tennessee. Journal of Health Care for the Poor and Underserved, v. 21, n. 1, p. 114-126, Feb, 2010.

REYNOLDS, D. Prostate cancer screening in African American men: barriers and methods for improvement. American Journal Men's Health, v. 2, n. 2, p. 172-177, Jun, 2008. ROBINSON, S. B.; ASHLEY, M.; HAYNES, M. A. Attitudes of African Americans regarding screening for prostate cancer. Journal Natl. Med. Assoc, v. 88, n. 4, p. 241-246, Apr, 1996.

ROBINSON, W. R. Obesity before Age 30 years and risk of advanced prostate cancer. American Journal of Epidemiology, v. 161, n. 12, p. 1107-1114, 2005.

ROOS, G; PRÄTTÄLÄ, R; KOSKI, K. Men, masculinity and food: interviews with Finnish carpenters and engineers. Appetite, v. 37, n. 1, p. 47-56, Aug. 2001.

ROSENSTOCK, I. M. Why people use health services. Milbank Memorial Fund, n. 44, p. 94-121, 1996.

SBU - Sociedade Brasileira de Urologia. Manual de boas práticas urológicas. 2009. Disponível em: < http://www. sbu. org. br. > Acesso em: 26 out. 2010.

SCHRÖDER, F. H. et al. Screening and prostate cancer mortality in a randomized european study. N Engl J Med, n. 360, p. 1320-1328, Mar, 2009.

SROUGI, M. Câncer de próstata: uma opinião médica. Sociedade Brasileira de Urologia (SBU), 1997. Disponível em: $<$ http://www. sbu. org. br. > Acesso em: 15 abr. 2010.

STEELE, C. B. et al. Knowledge, attitudes, and screening practices among older men regarding prostate cancer. American Journal Public Health, v. 90, n. 10, p. 1595-1600, Oct. 2000.

TALAVERA, G. A. et al. Predictors of digital rectal examination in U. S. Latinos. American Journal Prev. Med, v. 22, n. 1, p. 36-41, Jan. 2002.

TAYLOR, K. L. et al. Demographics, family histories, and psychological characteristics of prostate carcinoma screening participants. American Cancer Society, v. 85, n. 6, 1999. 
VOLK, R. J. et al. Patient education for informed decision making about prostate cancer screening: a randomized controlled trial with 1-year follow-up. Annals for Family Medicine, v. 1, n. 1, May/Jun. 2003.

YAN, Y. Y. Breast cancer: knowledge and perceptions of Chinese women in Hong Kong. Global Journal of Health Science, v. 1, n. 2, Oct. 2009.

WEINRICH, S. P. et al. Self-reported reasons men decide not to participate in free prostate cancer screening. ONF, v. 30, 2003. 